\title{
夏玉米不同部位干物质临界氮浓度稀释曲线的构建及对产量的估计
}

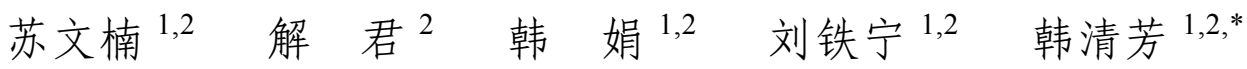

${ }^{1}$ 西北农林科技大学农学院 / 农业农村部西北黄土高原作物生理生态与耕作重点实验室, 陕西杨凌 $712100 ;{ }^{2}$ 中国旱区节水农业研究

院／西北农林科技大学旱区农业水土工程教育部重点实验室, 陕西杨凌 712100

摘 要: 准确和动态地沴断营养生长阶段植株氮状况, 对于评估植物氮需求、预测玉米产量以及优化氮素管理至关 重要。基于植物的氮诊断工具可优化夏玉米生产中氮素的管理, 本研究旨在开发和验证基于玉米地上部不同部位于 物质的临界氮浓度稀释曲线, 并建立玉米相对产量(relative yield, RY)与不同生长阶段氮素营养指数(nitrogen nutrition index, NNI)和累积氮素亏缺(accumulated nitrogen deficit, AND)的关系。本文以 2 个不同氮效率的品种为试验材料进 行连续 4 年的田间定位试验, 设置 4 个氮素水平 $\left(0 、 150 、 225\right.$ 和 $\left.300 \mathrm{~kg} \mathrm{hm}^{-2}\right)$, 分析不同施氮量对 2 个玉米品种营养 生长阶段干物质的影响, 基于叶干物质(leaf dry matter, LDM)、茎干物质(stem dry matter, SDM)和植物干物质(plant dry matter, PDM)，构建不同的临界氮浓度稀释曲线。结果表明，基于 LDM、SDM 和 PDM 建立的临界氮浓度稀释曲线，均 能很好地诊断玉米氮营养状况; 3 条临界氮浓度稀释曲线对产量进行预测比较发现, RY 与 NNI 和 AND 在不同生长阶 段之间的相关性均达到显著水平, 相关系数 $R^{2}$ 值均大于 0.65 , 其中 $R^{2}$ 值在 V12-VT 时期最大, 同时回归模型的验证 结果表明, 在 V12-VT 时期模型显示出可靠性。 $R^{2}$ 值大于 0.92 , RMSE 值小于 $10 \%$, 证实了模型在 V12 和 VT 两个时 期关系的稳定性。总的来说, 一定的条件下, 基于 LDM 和 SDM 建立的临界氮浓度稀释曲线可以对基于 PDM 建立的 临界氮浓度稀释曲线进行代替。在 V12-VT 阶段, RY 与 NNI 和 AND 的稳定关系很好地说明了在受氮素限制和非氮 素限制下 RY 的变化, 并对夏玉米产量进行准确的估计。本研究为花前玉米的氮肥管理提高粮食产量提供理论依据。 关键词: 临界氮浓度稀释曲线; 玉米; 氮营养指数; 累积氮素亏缺

\section{Construction of critical nitrogen dilution curve based on dry matter in diffe rent organs of summer maize and estimation of grain yield}

\author{
SU Wen-Nan ${ }^{1,2}$, XIE Jun ${ }^{2}$, HAN Juan ${ }^{1,2}$, LIU Tie-Ning ${ }^{1,2}$, and HAN Qing-Fang ${ }^{1,2, *}$ \\ ${ }^{1}$ College of Agronomy, Northwest A \& F University / Key Laboratory of Crop Physio-ecology and Tillage Science in North-western Loess Plateau, \\ Ministry of Agriculture and Rural Affairs / College of Agronomy, Northwest A\&F University, Yangling 712100, Shaanxi, China; ${ }^{2}$ Institute of Water \\ Saving Agriculture in Arid Areas of China / Key Laboratory of Agricultural Soil and Water Engineering in Arid and Semiarid Areas, Ministry of Edu- \\ cation, Northwest A\&F University, Yangling 712100, Shaanxi, China
}

\begin{abstract}
It is essential to accurate and dynamic diagnosis of plant nitrogen status at vegetative growth stage for the assessment of plant nitrogen demand and the prediction of crop yield as well as the optimization of nitrogen management in maize. Plant-based nitrogen diagnostic tool can be used to optimize nitrogen management in summer maize production. The aim of this study was to develop and verify critical nitrogen concentration dilution curves based on dry matter in different tissue of the plant, and to establish the relationship between relative yield (RY) nitrogen nutrition index (NNI), and accumulated nitrogen deficit (AND) at different growth stages in maize. We conducted a 4-year field study using four nitrogen application rates $(0,150,225$,
\end{abstract}

\footnotetext{
本研究由国家高技术研究发展计划(863 计划)项目(2013AA102902), 国家公益性行业(农业)科研专项(201303104)和国家自然科学基金项 目(31601256)资助。

This study was supported by the National High-Tech Research and Development Programs of China "863 Program" for the 12th Five-Year Plants (2013AA102902), the Special Fund for Agro-scientific Research in the Public Interest (201303104), and the National Natural Science Foundation of China (31601256).
}

*通信作者(Corresponding author): 韩清芳, E-mail: hanqf88@nwafu.edu.cn 第一作者联系方式: E-mail: asuwennan@163.com

Received (收稿日期): 2020-03-26; Accepted (接受日期): 2020-10-14; Published online (网络出版日期): 2020-11-03.

URL: https://kns.cnki.net/kcms/detail/11.1809.S.20201102.1154.003.html 
and $300 \mathrm{~kg} \mathrm{~N} \mathrm{hm}^{-2}$ ) and two maize cultivars (Zhengda 12 and Shaandan 609) to analyze the effects of nitrogen on dry matter at the vegetative growth stage, and based on leaf dry matter (LDM), stem dry matter (SDM), and plant dry matter (PDM), different critical nitrogen concentration dilution curves were developed. The results showed that the critical nitrogen concentration dilution curves based on LDM, SDM and PDM can well diagnose the nitrogen nutrition status of corn. The yield prediction results of three critical nitrogen concentration dilution curves showed that the relationship between RY and NNI, AND at different growth stages was highly significant, and the values of $R^{2}$ were all greater than 0.65 , where $R^{2}$ was the largest at V12-VT, and the verification of the regression model showed reliable model performance during the V12-VT period, with $R^{2}$ values greater than 0.92 and RMSE values less than $10 \%$, which confirmed the stability of the relationship between V12 and VT. Generally, under certain conditions, the critical nitrogen concentration dilution curve based on LDM and SDM can be used to replace the critical nitrogen concentration dilution curve based on PDM. The stable relationship between RY and NNI, RY and AND in V12-VT stage can well explain the change of RY under restricted and unrestricted nitrogen and estimate the yield of summer maize. This study provides the basis for nitrogen management of pre-anthesis to improve maize grain yield.

Keywords: critical nitrogen dilution curve; maize; nitrogen nutrition index; accumulated nitrogen deficit

氮肥对粮食增产的贡献率可达 $30 \% \sim 50 \%{ }^{[1]}$, 就 目前中国氮肥的使用现状，继续增加氮肥投入对提 高粮食产量的效果甚微, 并且降低了氮肥的利用效 率。同时, 调查结果显示, 约 $60 \%$ 70\%的氮肥通过 挥发和淋洗等途径有所损失 ${ }^{[2-3]}$, 这种现象在陕西关 中地区播前一次施入的施肥模式下变得更加严重。

因此, 针对作物不同生育阶段精确调控氮肥施用量, 对于增加作物产量, 提高氮肥利用效率和改善环境 问题具有重要战略意义 ${ }^{[4-5]}$ 。

进行植株氮营养状况的评估对于作物生长系统 的调查、监控、管理以及产量预测至为关键。评估 植株氮素营养状况的一些常用方式, 如叶绿素仪 ${ }^{[6]}$ 和遥感 ${ }^{[7]}$, 这些方法存在地域和年际间的不稳定性, 以及成本的问题导致在评估植株氮营养状况时受到 限制。临界氮浓度稀释曲线定义为最大作物生长所 需的最小氮浓度 ${ }^{[8]}$, 由于其在植物氮诊断中的准确 性和稳定性, 已引起全世界的广泛关注。随着研究 的深入, 国内外学者在构建方法和应用区域上对临 界氮浓度稀释曲线进行拓展 ${ }^{[9-12]}$ 。品种的更新(氮的 吸收和利用效率逐渐降低)和环境的变化以及模型 软件的发展, 对临界氮浓度稀释曲线参数提出更高 的要求。因此, 在不同部位干物质的基础上(而不是 仅仅传统上基于植株干物质(plant dry matter, PDM)) 开发临界氮浓度稀释曲线, 不仅可以更好地理解这 一概念, 而且有利于探清基因型和环境带来的作物 氮素动态的生理功能的差异, 同时适应模型软件的 发展。氮营养指数(nitrogen nutrition index, NNI)作为 氮素营养诊断的重要指标, 是用于基于临界氮浓度 稀释曲线的氮诊断的工具之一 ${ }^{[10,13-15]}$ 。NNI 这一概 念可以成功地区分植株的氮素营养状况, 并且将根 据这些曲线得出的诊断工具与作物模拟模型集成在 一起, 以协助作物氮素管理 ${ }^{[13-14,16-18]}$, 这些关系也
已用于估计作物对氮素的需求，以促进作物生长期 间的氮肥施用以及评估籽粒品质 ${ }^{[14]}$ 。水稻、小麦和 玉米营养生长期间 NNI 与相对产量(relative yield, RY) 的关系已用于估算籽粒产量 ${ }^{[17,19-20]}$ 。作物生长阶段对 于确定植物营养状况以预测玉米产量至关重要 ${ }^{[13,20]}$, 确定不同作物生长阶段 NNI 与 RY 的关系将有助于 更好地理解植株与氮的关系, 并有益于对作物氮的 动态建模, 因此, 需要进一步调查研究不同生育阶 段的 NNI 和相关氮参数是否可以预测玉米的产量。 例如 NNI 和累积氮亏缺 (accumulated nitrogen deficit, AND), 以量化植物中氮营养状态, 对氮素供应的响 应以及氮肥管理决策。

基于水稻 ${ }^{[21-24]}$ 和小麦 ${ }^{[25-26]}$ 的植物指数(叶、茎、 穗和叶面积指数)的氮浓度临界稀释曲线已经建立 并得到了验证。作为“源”器官的叶片, 不同生育阶段 由不同叶龄的叶片组成, 不同位置叶片的性质和功 能是不同的, 所以, 非破坏性取样且快速实时的单 叶监测冠层氮素浓度的氮营养诊断方式的好处被夸 大。营养阶段, 叶片作为功能结构, 由于其氮素的重 要生理功能, 基于整株植物叶片建立的临界氮浓度 稀释曲线, 可以更深入地了解作物氮素状态 ${ }^{[10]}$, 同 时, 茎作为结构组分, 其干物质对植物总干物质的 贡献显著高于叶片干物质, 因此, 它是整株植物临 界氮浓度稀释曲线的最主要决定因素。相关研究 ${ }^{[21]}$ 也指出基于水稻的茎干物质建立临界氮浓度稀释曲 线, 可以作为替代整株植物方法来评估植物氮的状 况从而用来建立施肥决策。同时, Zhao 等 ${ }^{[26]}$ 指出, 叶 片和茎为了满足各自代谢氮和结构氮的需求, 单独 建立作物叶片干物质和茎干物质的临界氮浓度稀释 曲线非常重要, 这也将有利于作物模拟模型参数的

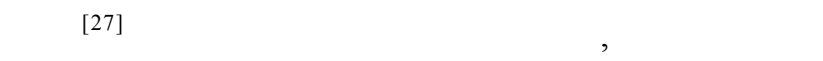
法进行综合研究, 可以对植物氮的状况提供有用的 
见解。现有的研究大多集中于使用地上部干物质和 叶片干物质建立稀释曲线，尽管基于其他部位的稀 释曲线得到了建立, 但数量有限, 在玉米上更是少 数。在陕西夏玉米主栽区, 对于临界曲线的研究仅 局限于基于整株玉米干物质基础上建立的曲线 ${ }^{[28-29]}$, 基于不同部位干物质的临界氮浓度稀释曲线的系统 研究还未见报道, 本试验是对临界氮浓度稀释曲线 在本生态区的验证和补充。此外，作物生长期间的不 同器官干物质分配和氮素分布, 对于理解实现作物 最大产量的氮肥管理及其限制因素具有重要作用。所 以，对于研究基于不同部位干物质的临界氮浓度稀 释曲线之间的联系, 以测试作物氮稀释理论在玉米 开花前期诊断玉米氮素状况的适用性上还需试验数 据来阐明。玉米氮素的诊断能够及时掌握各生育时期 的营养状况, 因此, 要准确评估作物的氮素状况, 需 要在作物关键生长阶段进行作物的氮素评估, 并以 此确定科学合理的施肥决策。综上, 本研究利用不同 氮效率夏玉米品种, 开发基于不同部位干物质的临界 氮浓度稀释曲线，以确定它们的差异。在此基础上，采 用作物关键生长阶段 NNI 和 AND 对玉米 RY 进行估 算, 在西北气候条件下, 评估玉米的氮素营养状况, 预测产量。这项研究代表了对玉米干物质基础氮浓度 稀释曲线的首次最全面的研究, 将为诊断植物氮素状 况提供综合方法, 并为玉米关键生长阶段精确氮素管 理提供指导, 从而为玉米产量估算提供有用的方法。

\section{1 材料与方法}

\section{1 试验设计}

试验在西北农林科技大学试验基地进行。该试 验基地位于陕西省杨凌 $\left(34^{\circ} 20^{\prime} \mathrm{N}, 108^{\circ} 24^{\prime} \mathrm{E}\right.$, 海拔 $454.8 \mathrm{~m})$, 属于暖温带季风半湿润气候区。近 20 年 的年平均气温为 $13.5^{\circ} \mathrm{C}$, 每年的总日照时间为 $2196 \mathrm{~h}$, 该地点的年平均降水量为 $580.5 \mathrm{~mm}$, 年平均蒸发量 为 $993.2 \mathrm{~mm}$ 。 2014 年 6 月至 2017 年 10 月, 连续 4 个玉米季进行试验。试验地前茬作物为冬小麦, 试 验地土壤类型为耧土, 耕层土壤化学性质为: 有机 质 $14.3 \mathrm{~g} \mathrm{~kg}^{-1}$ 、全氮 $1.09 \mathrm{~g} \mathrm{~kg}^{-1}$ 、有效磷 $9.4 \mathrm{mg} \mathrm{kg}^{-1}$ 和速效钾 $127 \mathrm{mg} \mathrm{kg}^{-1}$ 。供试品种为正大 12 (氮高效 品种, Zhengda 12, ZD)和陕单 609 (氮低效品种, Shaandan 609, SD)。试验设计为随机区组设计, 每年 每个处理 3 个重复, 小区面积 $39 \mathrm{~m}^{2}$, 株距 $60 \mathrm{~cm}$, 行 距 $25 \mathrm{~cm}$ 。设置 4 个施氮水平, 纯氮施用量分别为 0 (N0)、150 (N150)、225 (N225)和 $300 \mathrm{~kg} \mathrm{hm}^{-2}$ (N300)。
$1 / 2$ 纯氮在玉米播种前施入 (播前 $5 \mathrm{~d}$ ), 剩余 $1 / 2$ 纯氮 在玉米大喇叭口期开沟追施。磷和钾肥在播种前一 次性施入, 用量均为 $150 \mathrm{~kg} \mathrm{hm}^{-2}$ 。氮肥为尿素(含 $\mathrm{N}$ $46.4 \%$ ), 磷肥为过磷酸钙 (含 $\mathrm{P}_{2} \mathrm{O}_{5} \quad 12.0 \%$ ), 钾肥为氯 化钾(含 $\mathrm{K}_{2} \mathrm{O} 60.0 \%$ )。

玉米播种时间分别为 2014 年 6 月 12 日、2015 年 6 月 12 日、2016 年 6 月 17 日和 2017 年 6 月 15 日, 在三叶期定苗为 67,500 株 $\mathrm{hm}^{-2}$ 。玉米收获时间 分别为 2014 年 10 月 14 日、2015 年 10 月 15 日、2016 年 10 月 3 日和 2017 年 10 月 14 日。田间管理与当地 保持一致, 三至五叶期进行化学除草, 拔节期防治病 虫害。试验在大喇叭口期以 $75 \mathrm{~mm}$ 的水量进行灌溉。

\section{2 测定项目及方法}

在玉米的 V3、V6、V8、V12、VT 和 R6 时期[分 别代表玉米的三叶期、六叶期(拔节期)、八叶期、十 二叶期(大喇叭口期)、抽雄期和完熟期], 4 年对应的时 间在表 1 中呈现。每个小区选择具有 3 5 株玉米, VT 之前植株分为叶片和茎 2 个部分, VT 之后, 植株分为 叶片、茎、穗轴、苞叶和籽粒, 所有部分在 $105^{\circ} \mathrm{C}$ 杀青 $30 \mathrm{~min}$, 然后在 $70^{\circ} \mathrm{C}$ 烘干至恒重, 分别称重, 记录叶片、 茎和植株的总质量。粉碎过 100 目篮后, 称取 $0.02 \mathrm{~g}$ 样 品, 采用半微量凯氏定氮法测定各部位的氮浓度 ${ }^{[30]}$ 。

玉米收获时，每个小区随机收获 30 个玉米进行 晾晒和脱粒, 测定籽粒产量, 并按照国家玉米籽粒 入库标准(含水量 $14 \%$ )折算产量。

\section{3 基于各部位干物质临界氮浓度稀释曲线的} 建立

根据 Justes ${ }^{[8]}$ 的方法, 计算临界氮浓度稀释曲线 的步骤如下: (1)利用单因素方差分析, 对不同氮水 平的地上部植株干物质(叶片干重和茎干重)分为作 物生长受氮素限制组和氮充足作物生长不受氮素限 制组; (2)对受氮素限制处理的干物质与氮浓度进行 线性拟合, 对不受氮素限制处理的干物质取平均值 并通过平均值做垂直于横轴的垂线, 2 条直线的交点 为临界氮浓度; (3)拟合干物质与对应临界氮浓度的 散点图, 基于幂回归模型(Freundlich 模型)的异速函 数用于确定观察到的氮浓度降低与叶片、茎和植株 干物质(LDM, SDM 和 PDM)增加之间的关系。玉米 临界氮浓度稀释曲线模型为:

$$
N_{\mathrm{c}}=a \mathrm{DM}^{-b}
$$

式中, $N_{\mathrm{c}}$ 代表作物临界氮含量, $\mathrm{g} \mathrm{kg}^{-1}$; DM 为地上部干 物质, $\mathrm{t} \mathrm{hm}^{-2}$; 参数 $a$ 代表干物质为 $1 \mathrm{t}$ 时的临界氮浓度, 参数 $b$ 为临界氮浓度稀释曲线斜率的统计学参数。 
表 $12014-2017$ 年干物质取样时期

Table 1 Dry matter sampling period from 2014 to 2017

\begin{tabular}{clccccc}
\hline 项目 Item & V3 (M/D) & V6 (M/D) & V8 (M/D) & V12 (M/D) & VT (M/D) & R6 (M/D) \\
\hline 2014 & $6 / 27$ & $7 / 12$ & $7 / 17$ & $7 / 30$ & $8 / 13$ & $10 / 14$ \\
2015 & $6 / 29$ & $7 / 15$ & $7 / 20$ & $8 / 2$ & $8 / 17$ & $10 / 15$ \\
2016 & $7 / 1$ & $7 / 14$ & $7 / 21$ & $8 / 15$ & $10 / 3$ \\
2017 & $6 / 29$ & $7 / 13$ & $7 / 21$ & $8 / 4$ & $8 / 13$ & $10 / 14$ \\
\hline
\end{tabular}

V3、V6、V8、V12、VT 和 R6 分别表示玉米的三叶期、六叶期(拔节期)、八叶期、十二叶期(大喇叭口期)、抽雄期和完熟期。

V3, V6, V8, V12, VT, and R6 represent the third, sixth, eighth, twelfth leaf stages, tasseling and physiological maturity stages, respectively. $\mathrm{M} / \mathrm{D}:$ month/day.

\section{4 临界氮浓度稀释曲线模型的验证}

为了校验模型的精度, 本研究通过计算标准化 均方根误差 $(n-\mathrm{RMSE})$ 来评判模型的精度:

$$
\begin{aligned}
& \mathrm{RMSE}=\sqrt{\frac{\sum_{i=1}^{n}\left(P_{i}-O_{i}\right)^{2}}{n}} \\
& n-\mathrm{RMSE}=\frac{\mathrm{RMSE}}{S} \times 100 \%
\end{aligned}
$$

模型的验证采用标准化均方根误差 $n$-RMSE ${ }^{[31]}$ 来检测模型的拟合度。

式中: $P_{i}$ 和 $O_{i}$ 分别为临界氮测定值和模拟值; $n$ 为样 本量; $S$ 为实测数据的平均值。 RMSE 值越小, 模拟 值与测定值的一致性越好, 偏差越小, 即模型的预 测精度越高。Jamieson 等 ${ }^{[32]}$ 认为: $n-\mathrm{RMSE}<10 \%$, 模 型稳定性极好; $10 \%<n-\mathrm{RMSE}<20 \%$, 模型稳定性较好; $20 \%<n-\mathrm{RMSE}<30 \%$ ，模型稳定性一般； $n$-RMSE $>$ $30 \%$, 模型稳定性较差。

最大氮曲线 $\left(N_{\max }\right)$ 通过使用不受氮素限制的数据 点建立(2014 年和 2015 年的 N300 处理), 最小氮曲线 $\left(N_{\min }\right)$ 通过使用受氮素限制处理的数据点建立。2016 和 2017 年的试验数据用于验证 $N_{\mathrm{c}} 、 N_{\max }$ 和 $N_{\min }$ 曲线 ${ }^{[7]}$ 。

1.5 氮营养指数, 氮亏缺相对产量和氮效率的计算

为了更精确地反映玉米植株氮素是否适宜, 氮 素营养指数(NNI)被用来评估玉米生育期内的氮素 状况。

$$
\mathrm{NNI}=\frac{N_{\mathrm{a}}}{N_{\mathrm{c}}}
$$

式中, $N_{\mathrm{a}}$ 为地上部氮浓度实测值, $N_{\mathrm{c}}$ 为地上部氮浓度 的临界值。当 NNI = 1 时氮营养状况为最适的, 当 $\mathrm{NNI}>1$, 氮营养处于过剩的状况, 反之, 当 $\mathrm{NNI}<1$ 时，植株处于营养不足的状态。

根据式(4)可以推出玉米氮积累亏缺(AND $)^{[18]}$ :

$$
\mathrm{AND}=N_{\mathrm{cna}}-N_{\text {na }}
$$

式中, AND 为氮亏缺量 $\left(\mathrm{kg} \mathrm{hm}^{-2}\right), N_{\mathrm{cna}}$ 为临界氮浓度 下作物氮素积累量 $\left(\mathrm{kg} \mathrm{hm}^{-2}\right), N_{\mathrm{na}}$ 为作物实际氮素积
累量 $\left(\mathrm{kg} \mathrm{hm}^{-2}\right)$ 。当 $\mathrm{AND}=0$ 时, 证明作物体内氮素 最佳, 当 AND $>0$ 时, 说明氮积累不足, 当 AND $<0$ 时, 说明氮素积累过量。

$$
\text { 相对产量 }(\mathrm{RY})=\text { 籽粒产量/最高产量 }
$$

氮肥偏生产力 (partial factor productivity nitrogen, PFPN) $=$ 籽粒产量/施氮量

氮肥回收率 (recovery efficiency of nitrogen, $R E N)=($ 施氮植株含氮量-不施氮植株含氮量 $) /$ 施氮量

氮素利用效率 (nitrogen utilization efficiency, NutE) $=$ 籽粒产量/植株氮素积累量

氮素吸收效率(nitrogen uptake efficiency, NupE) $=$ 植株氮素积累量/(土壤氮 + 施氮量 $)$

\section{6 产量预测模型的建立和验证}

对 4 年的试验数据进行方差分析, 用 2014 年和 2015 年的试验数据分别对 NNI 和 AND 与 RY 每个 时期进行回归分析, 2016 年和 2017 年获得的试验数 据对以上回归模型进行验证。根据回归分析结果, 将 4 年的数据进行汇总, 以建立最终产量预测模型。 预测值和观察值之间的决定系数 $\left(R^{2}\right)$, 均方根误差 (RMSE) 和标准化均方根误差 $n-\mathrm{RMSE}$ 用于检验 NNI, AND 和 RY 之间的回归模型的拟合度。

\section{7 数据分析}

每个采样期, 年份和品种的数据, 用 SPSS 18.0 进行方差分析(SPSS Inc., Chicago, IL, USA)。采用最 小显著差异法进行处理间的比较, 显著水平为 0.05 。 $N_{\mathrm{c}}$ 曲线回归分析使用 Microsoft Excel 2013 进行。用 SPSS 18.0 对 RY 和 NNI 与 RY 和 AND 进行线性加 平台分析。

\section{2 结果与分析}

2.1 基于不同部位临界氮浓度稀释曲线构建及 临界氮浓度常数的确定

4 年的试验结果表明, 2 个玉米品种的氮效率存 在显著差异(表 2), 除 2017 年氮肥回收效率和 2015 


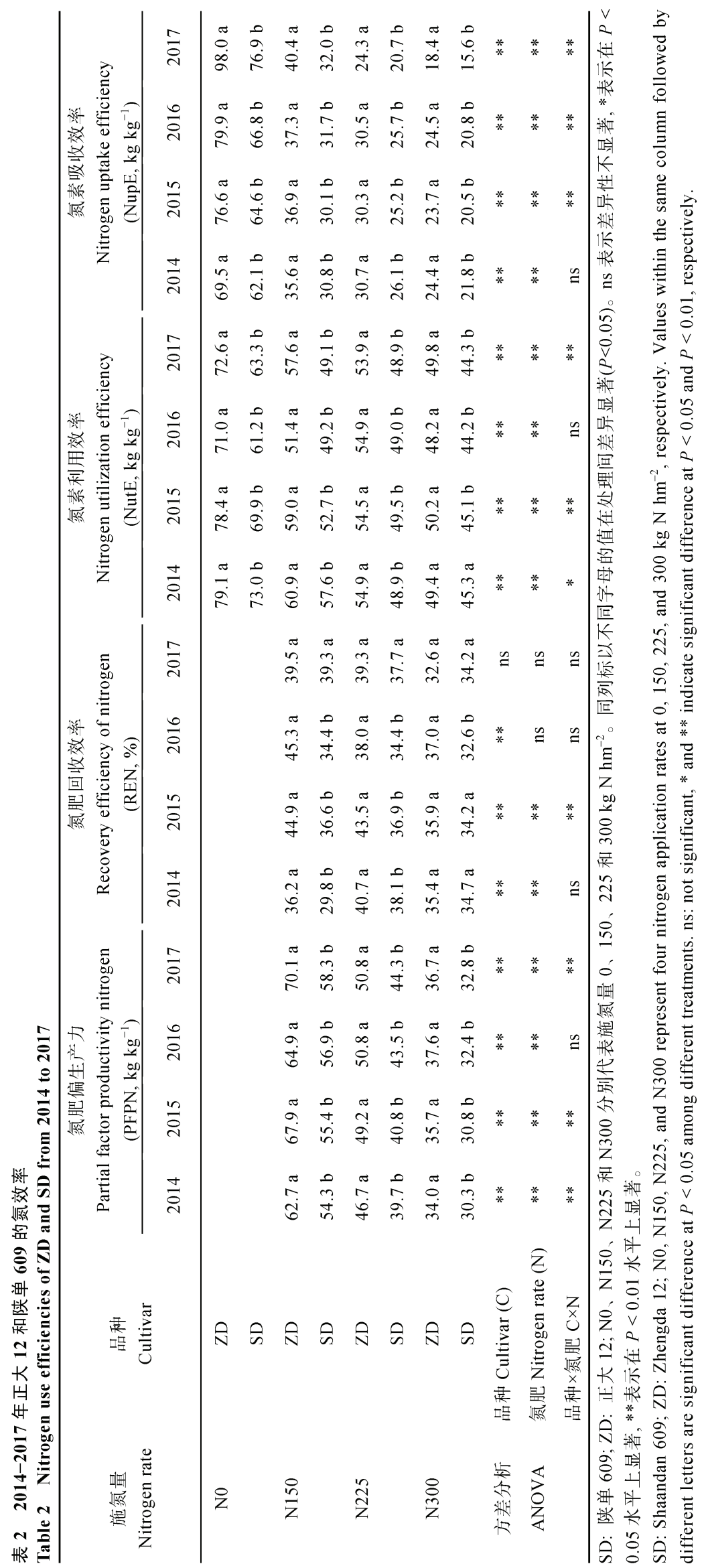




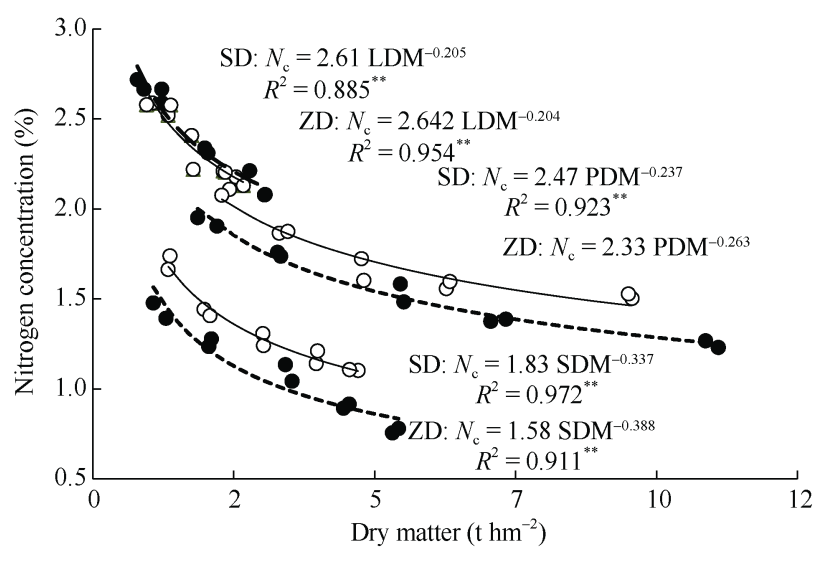

图 1 基于叶片、茎和植株干物质建立的 2 个玉米品种临界氮浓 度稀释曲线的差异比较

Fig. 1 Comparison of critical $\mathrm{N}$ dilution curves of two maize hybrids on different bases (leaf dry matter basis, stem dry matter basis, and plant dry matter basis) with varied nitrogen rates $\mathrm{SD}$ ：陕单 609; ZD：正大 $12 ; N_{\mathrm{c}}$ ：地上部氮浓度的临界值; LDM: 叶片干物质; SDM: 茎干物质; PDM: 植株干物质。“○”表示品种 陕单 609, “・”表示品种正大 12 ; “一”表示品种陕单 609, , - - ”表 示品种正大 12 。 ${ }^{* *}$ 表示在 $P<0.01$ 水平上显著。

SD: Shaandan 609; ZD: Zhengda 12; $N_{\mathrm{c}}$ : critical value of shoot nitrogen concentration; LDM: leaf dry matter basis; SDM: stem dry matter basis; PDM: plant dry matter. Shaandan 609 is marked by "०", Zhengda 12 is marked by "•"; "—- means the curves of Shaandan 609, “- . -" means the curves of Zhengda $12 .^{* *}$ indicates significant difference at $P<0.01$.

年 N 300 处理外，不同年际, 2 个玉米品种氮效率差 异均达到显著水平, $\mathrm{ZD}$ 的氮效率显著高于 $\mathrm{SD}$ 。本 试验中, $\mathrm{ZD}$ 被定义为氮高效品种, $\mathrm{SD}$ 被定义为氮 低效品种。在本研究中, 用 2014 年和 2015 年试验 数据, 对 2 个品种分别基于叶片、茎和植株干物质 (LDM、SDM 和 PDM)建立了临界氮浓度稀释曲线。 从图 1 中可以看出, 建立基于叶片、茎和植株干物 质的临界氮浓度模型的数据, 适宜叶片, 茎和植株 干物质的范围分别是 $0.76 \sim 2.93 、 1.03 \sim 5.14$ 以及 $1.78 \sim 10.59 \mathrm{t} \mathrm{hm}^{-2}$ 。基于叶片、茎和植株干物质建立 的临界氮浓度曲线的参数 $a$ 和 $b$ 值的范围分别是 $1.58 \sim 2.64$ 和 $0.204 \sim 0.388$ 。其中，对于参数 $a$ 来说，基
于叶片干物质的临界氮浓度稀释曲线的 $a$ 值最大, 最小的是茎干物质临界氮浓度稀释曲线的 $a$ 值; 而 对于参数 $b$ 来说, 正相反, 基于茎干物质的临界氮 浓度稀释曲线的值最大，而基于叶片干物质的临界 氮浓度稀释曲线的值最小。2 个品种基于 LDM、SDM 和 $\mathrm{PDM}$ 临界氮浓度稀释曲线的 $R^{2}$ 的范围为 0.86 0.98。对 2 个品种分别进行临界氮浓度稀释曲 线的拟合, 'ZD'方程为: $N_{\mathrm{c}}=2.64 \mathrm{LDM}^{-0.204} 、 N_{\mathrm{c}}=1.58$ $\mathrm{SDM}^{-0.388} 、 N_{\mathrm{c}}=2.33 \mathrm{PDM}^{-0.263}$; 'SD'方程为: $N_{\mathrm{c}}=2.61$ $\mathrm{LDM}^{-0.205} 、 N_{\mathrm{c}}=1.83 \mathrm{SDM}^{-0.337} 、 N_{\mathrm{c}}=2.47 \mathrm{PDM}^{-0.237}$ 。 为进一步分析基于不同部位建立的临界氮浓度稀释 曲线 2 个品种的差异, 将曲线直线化处理, 采用协 方差分析方法 ${ }^{[33]}$, 得到结果, 2 个品种叶片斜率和截 距得到的 $P$ 值大于 0.05 , 而茎和植株斜率和截距得 到的 $P$ 值都小于 0.05 , 说明除叶片外, 基于茎和植 株干物质建立的临界氮浓度稀释曲线在品种间存在 显著差异。

\section{2 临界氮浓度稀释曲线的验证}

选取 2016 年和 2017 年数据对临界氮浓度曲线 进行验证。结果表明, $N_{\mathrm{c}}$ 稀释曲线可以区分玉米受 氮素限制和不受氮素限制的生长条件。受氮素限制 处理的所有数据点基本上都落在 $N_{\mathrm{c}}$ 稀释曲线的下方, 不受氮素限制处理的数据点落在 $N_{\mathrm{c}}$ 稀释曲线上或者 在其之上(图 2)。同时, 校验模型的精度, 其步骤为: 将实测干物质数据点分别带入式(1)中计算临界氮含 量模拟值, 将模拟值分别与观测值比较(表 3)。氮高 效品种 ZD 基于叶片、茎和植株模型偏差分别为 $1.8 \% 、 16.7 \%$ 和 $9.1 \% ，<20 \%$ 模型稳定性极好或较好, 氮低效品种 SD 偏差分别为 $1.4 \% 、 3.5 \%$ 和 $5.0 \%$, $<10 \%$ 模型稳定性极好。可见，所构建的 2 个品种的 临界氮浓度稀释曲线模型具有很好的精度，表明本 研究所建立的不同氮效率品种基于各部位的临界氮 浓度稀释曲线可进一步用于植株的氮营养诊断。

表 3 临界氮浓度稀释曲线的验证

Table 3 Calibration of critical $N$ dilution curve basis on leaf dry matter, stem dry matter and plant dry matter in maize

\begin{tabular}{ccccc}
\hline 参数 \\
Parameter
\end{tabular}



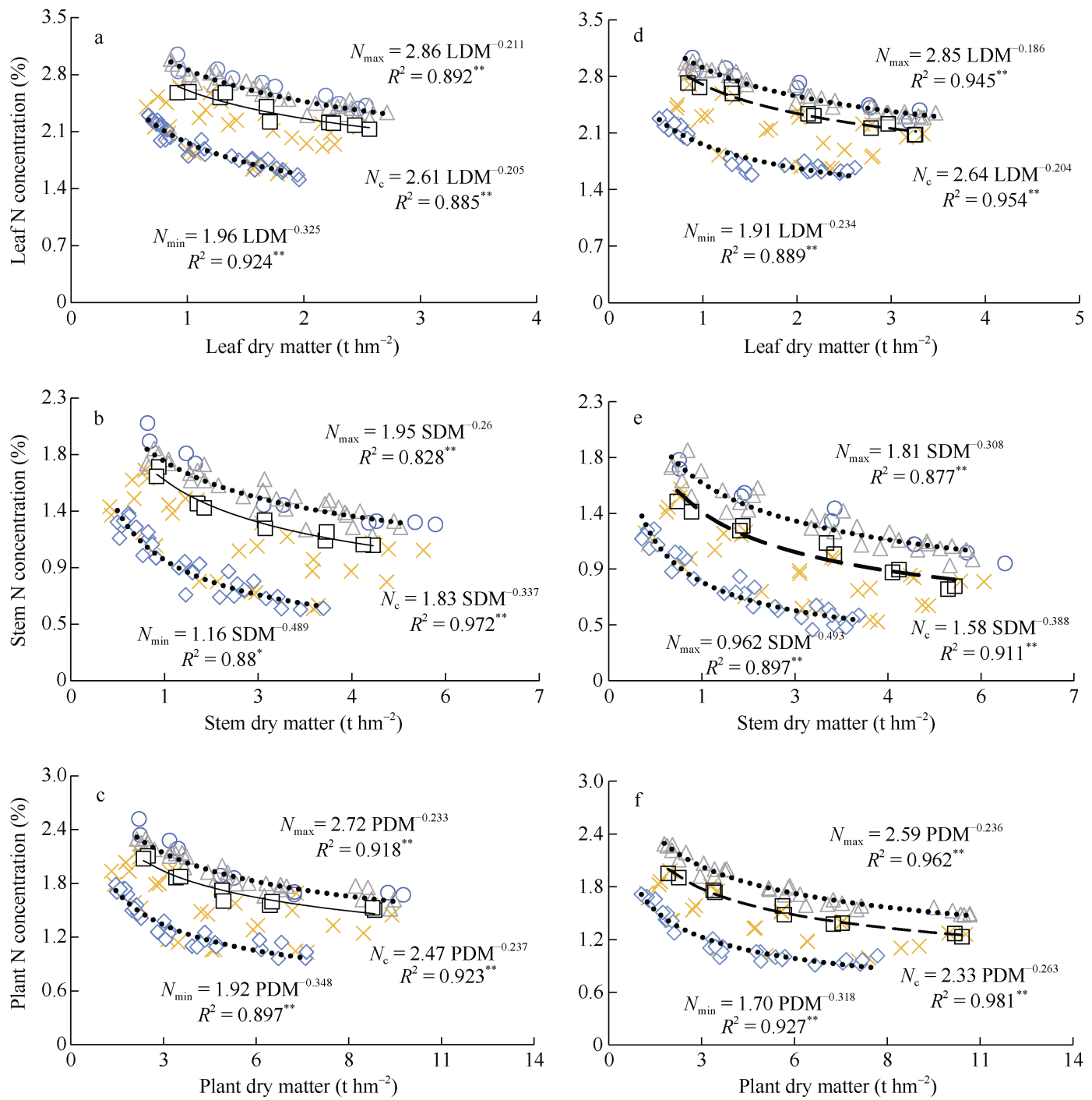

图 2 用 2016-2017 年获得的数据验证 $N_{\mathrm{c}}$ 稀释曲线

Fig. 2 Validation of the $\mathrm{N}$ c dilution curve using data from experiments performed from 2016 to 2017

符号(○)和( $\times$ )分别代表 2016 年和 2017 年的不受氮素限制值和受氮素限制值。a、b 和 c 实线分别代表陕单 609 的叶片、茎和植株的 $N_{\mathrm{c}}$ 稀释曲线, $\mathrm{d}$ 、 $\mathrm{e}$ 和 $\mathrm{f}$ 实线分别代表正大 12 的叶片、茎和植株的 $N_{\mathrm{c}}$ 稀释曲线。两侧的虚线代表最小和最大的曲线, 用 $2014-2015$ 年 不受氮素限制值 $(\triangle)$ 和受氮素限制值 $(\diamond)$ 获得。 $N_{\min }$ 和 $N_{\max }$ 为氮浓度最小和最大值; LDM: 叶片干物质; SDM: 茎干物质; PDM: 植株干 物质。 ${ }^{* *}$ 表示在 $P<0.01$ 水平上显著。

The symbols $(\circ)$ and $(\times)$ represent non-N-limiting and N-limiting values from 2016 to 2017, respectively. The solid curved lines a, b, and c represent the $N_{\mathrm{c}}$ dilution curves of the leaf, stem, and plant of Shandan 609, respectively, and the solid curved lines d, e, and f represent the $N_{\mathrm{c}}$ dilution curves of the leaf, stem, and plant of Zhengda 12, respectively. The dotted lines on either side represent the curves for the minimum limits, which are developed using data from N-limiting $(\diamond)$ and non-N-limiting $(\triangle)$ treatments from 2014 to $2015 . N_{\min }$ and $N_{\max }$ are minimum and maximum of nitrogen concentration; LDM: leaf dry matter basis; SDM: stem dry matter basis, PDM: plant dry matter. ${ }^{* *}$ indicates significant difference at $P<0.01$.

\section{3 基于不同部位临界氮浓度稀释曲线氮营养} 指数和相对产量, 氮亏缺和相对产量的关系

$\mathrm{NNI}$ 与 RY, AND 与 RY 之间的关系如图 3 图 5 所示，随着 NNI 的增加, RY 均呈现线性的增长趋势, 直到 RY 不随 NNI 的增加为止, 其变化趋势呈现出 线性加平台模式。相反, 随着 AND 的增加, RY 均呈 现先不变后线性降低的趋势。基于叶片干物质的临 界氮浓度稀释曲线, NNI 与 RY 之间的决定系数范围
为 $0.961 \sim 0.997$, AND 与 RY 之间的决定系数范围为 0.920 0.997, 基于茎干物质的临界氮浓度稀释曲线, NNI 与 RY 之间的决定系数范围为 $0.956 \sim 0.995$, AND 与 RY 之间的决定系数范围为 $0.919 \sim 0.991$, 基 于植株干物质的氮临界曲线, NNI 与 RY 之间的决定 系数范围为 0.913 0.992, AND 与 RY 之间的决定系 数范围为 $0.969 \sim 0.996$ 。因此, 可以从作物营养生长 的氮营养状态评价对产量的影响。 

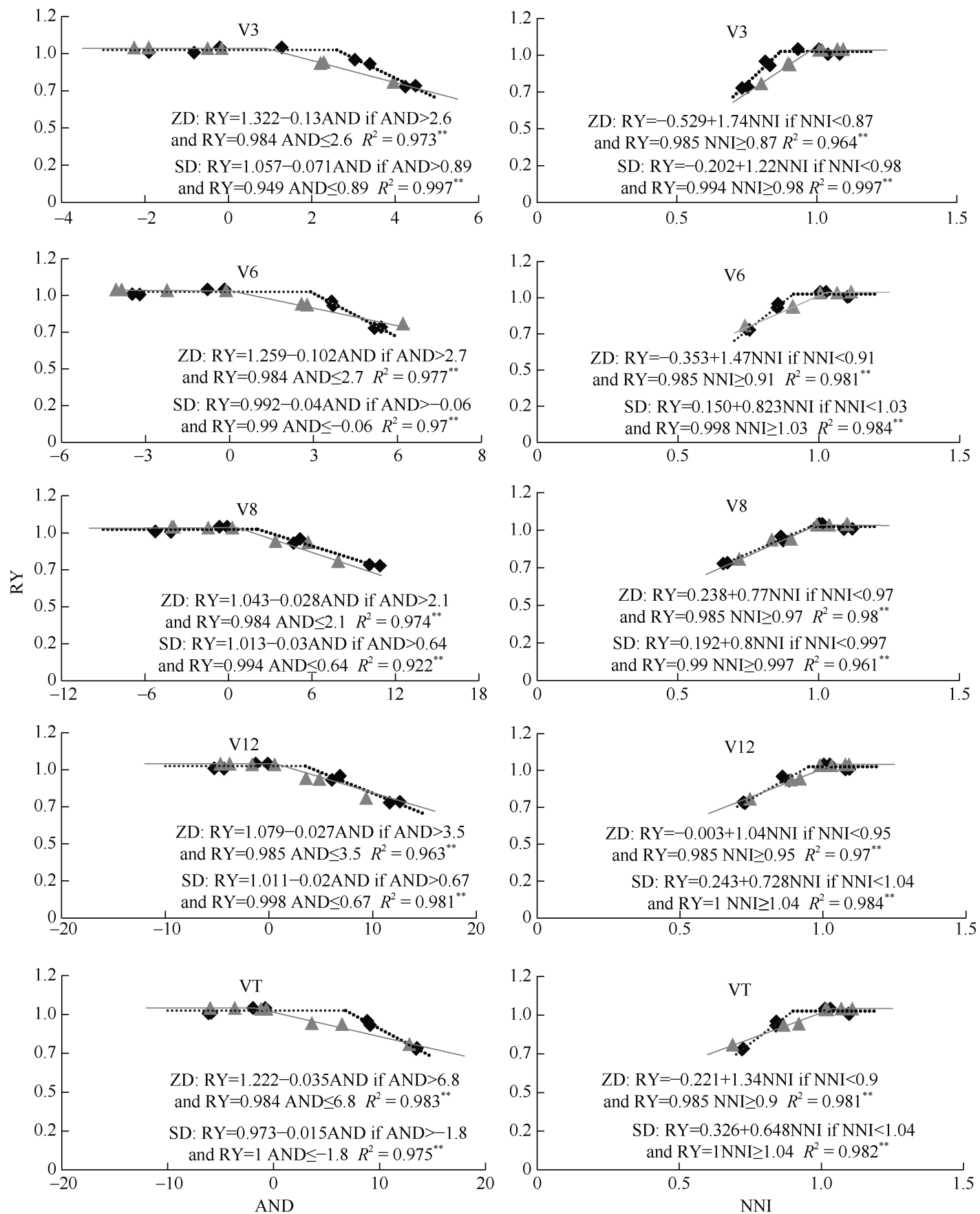

图 3 基于叶片干物质建立的临界氮浓度曲线得到的 RY 与 NNI 和 AND 的关系

Fig. 3 Relationships between relative yield (RY) and the nitrogen nutrition index (NNI), relative yield (RY) and the accumulated nitrogen deficit (AND) of two maize hybrids with varied $\mathrm{N}$ rates on the base of leaf dry matter $\mathrm{N}$ dilution curves

V3、V6、V8、V12、VT 和 R6 分别代表玉米的三叶期、六叶期(拔节期)、八叶期、十二叶期(大喇叭口期)、抽雄期和完熟期。SD: 陕单 609; ZD: 正大 12 ; “ $\triangle$ ”表示品种陕单 609 , “ $\triangleright$ ”表示品种正大 12 ; “一”表示品种陕单 609, “......”表示品种正大 12 。”表示在 $P<0.01$ 水平上显著。 V3, V6, V8, V12, VT and R6 represent the third, sixth, eighth, twelfth leaf stages, tasseling and physiological maturity stages, respectively. SD: Shaandan 609; ZD: Zhengda 12; Shaandan 609 is marked by " $\triangle$ ", Zhengda 12 is marked by " $\triangle$ "; "—” means the curves of Shandan 609, “....." means the curves of Zhengda $12 .{ }^{* *}$ indicates significant difference at $P<0.01$.

\section{4 相对产量模型的验证和最终模型}

$\mathrm{NNI}$ 与 RY 之间关系的 RMSE 变化范围为 $0.03 \sim 0.12, n-\mathrm{RMSE}$ 的变化范围为 $2.82 \sim 13.7, R^{2}$ 的变 化范围为 $0.76 \sim 0.99$ 。AND 与 RY 之间的 RMSE 的
变化范围为 $0.06 \sim 0.13, n-\mathrm{RMSE}$ 的变化范围为 $3.55 \sim 14.84, R^{2}$ 的变化范围为 $0.65 \sim 0.98$ (表 4)。 NNI 和 RY 的关系及 AND 和 RY 的关系, 在 V12 和 VT 两个时期基于叶片、茎和植株氮稀释模型建立的回 

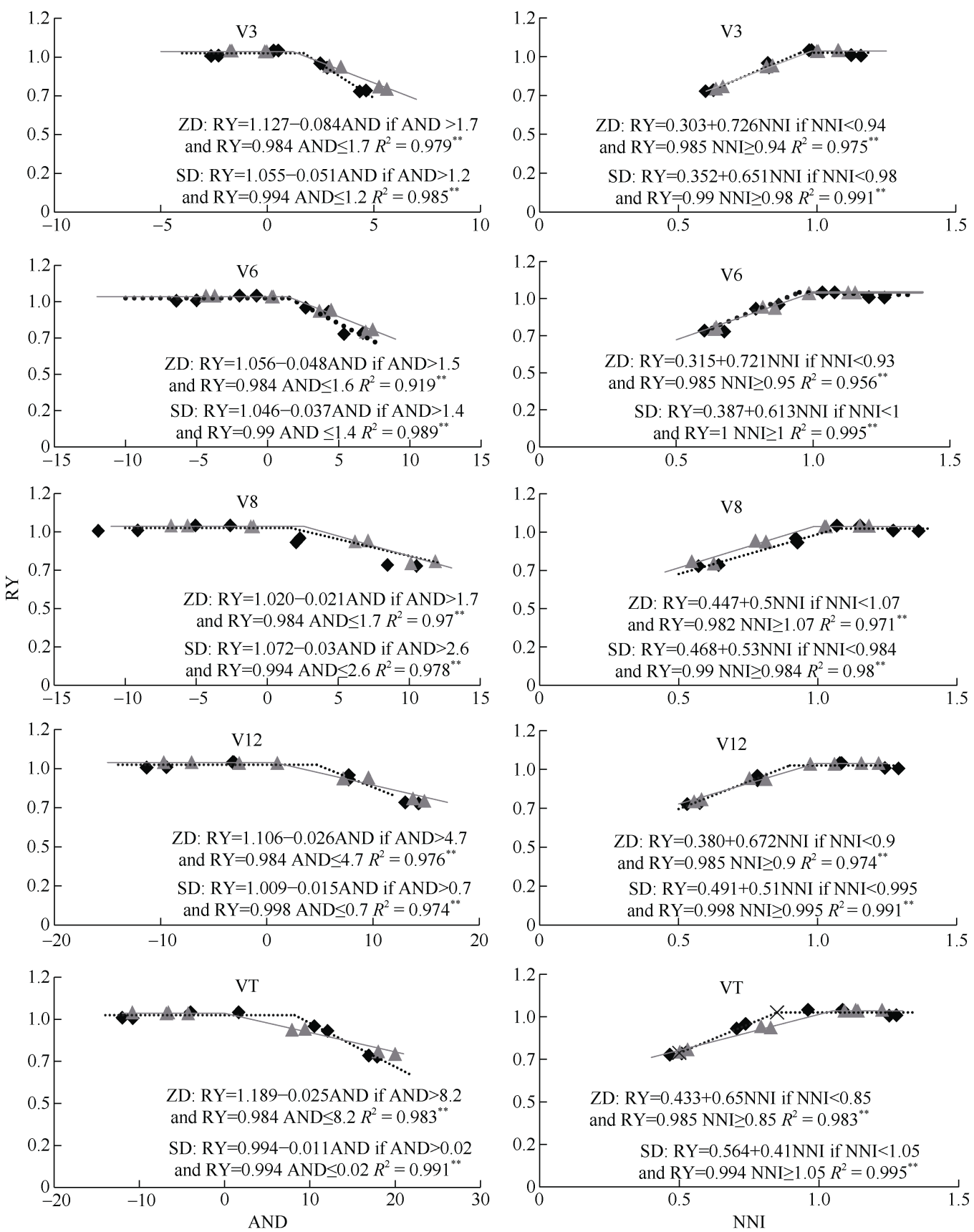

图 4 基于茎干物质建立的临界氮浓度曲线得到的 RY 与 NNI 和 AND 的关系

Fig. 4 Relationships between relative yield (RY) and the nitrogen nutrition index (NNI), relative yield (RY) and the accumulated nitrogen deficit (AND) of two maize hybrids with varied $\mathrm{N}$ rates on the bases of stem dry matter $\mathrm{N}$ dilution curves 缩写和符号同图 3。 ${ }^{* *}$ 表示在 $P<0.01$ 水平上显著。

Abbreviations and symbols are the same as those given in Fig. $3 .^{* *}$ indicates significant difference at $P<0.01$.

归关系 RMSE 和 $n-\mathrm{RMSE}$ 在 2 个品种中较小, 同时 $R^{2}$ 较大, 综合来看, 这 2 个时期模型的稳定性最强, 在 NNI 与 RY 之间和 AND 与 RY 之间的关系对玉米 生产中产量的估计具有实际意义。基于 NNI 与 RY 和 AND 与 RY 关系的精度和准确度的综合考虑, 对 V12 和 VT 两个时期建立相对产量模型(表 5), $R^{2}>0.941$ 。

\section{3 讨论}

近 20 年来, 临界氮浓度稀释曲线一直被用于作 物的氮精确管理。在不同气候条件下, 在 LDM、 SDM、穗干物质和 PDM 基础上建立了临界氮浓度

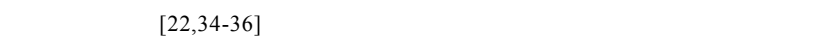
建立临界氮浓度稀释曲线与现有这些曲线进行比较 

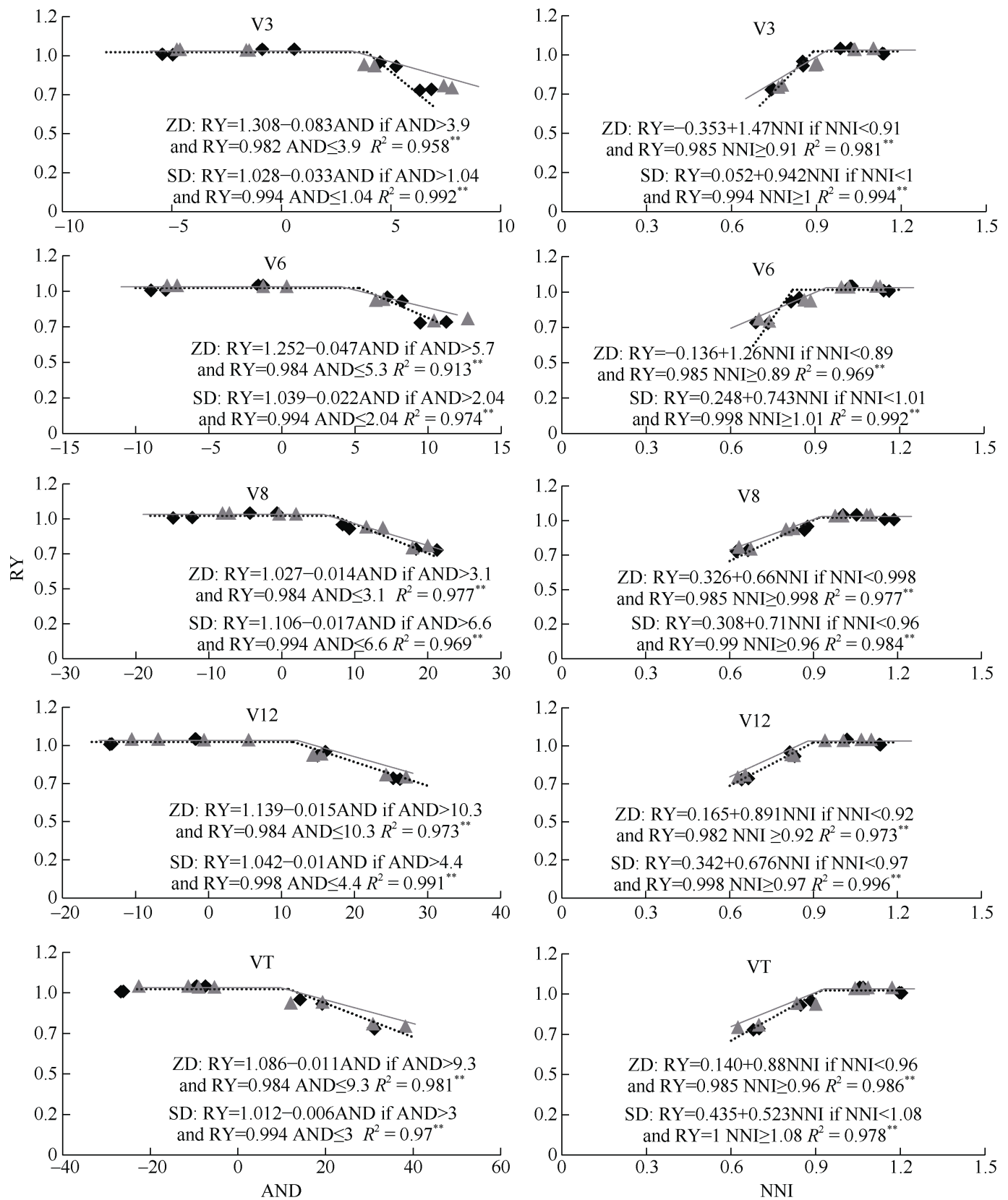

图 5 基于植株干物质建立的临界氮浓度曲线得到的 RY 与 NNI 和 AND 的关系

Fig. 5 Relationships between relative yield (RY) and the nitrogen nutrition index (NNI), relative yield (RY) and the accumulated nitrogen deficit (AND) of two maize hybrids with varied $N$ rates on the bases of plant dry matter $N$ dilution curves 缩写和符号同图 3。 ${ }^{* *}$ 表示在 $P<0.01$ 水平上显著。

Abbreviations and symbols are the same as those given in Fig. 3 . $^{* *}$ indicates significant difference at $P<0.01$.

来评估玉米的氮营养状况，以深入了解这一概念， 然后找到最合适的估算氮营养方法同时建立产量预 测模型。

3.1 基于不同部位建立的临界氮浓度稀释曲线 之间的对比及与其他模型的比较

与之前玉米、小麦和水稻作物上基于不同部位 建立的氮浓度稀释曲线一致 ${ }^{[9,19,34]}$, 基于 LDM 和 $\mathrm{SDM}$ 的临界氮浓度稀释曲线与基于 PDM 的模型建
立基本一致。2 个品种各部位的临界氮浓度稀释曲 线的决定系数均达到显著水平，在不同年份间具有 很好的稳定性，因此，可以用于对关中地区夏玉米 氮素的诊断方法。在作物中使用不同的植物指数和 氮浓度的关系, 而不是单独使用 PDM, 有利于增进 对临界氮浓度稀释曲线概念的理解 ${ }^{[22]}$ 。对本文建立 的基于不同部位的临界氮浓度稀释曲线和已经发表 的不同指标下的临界氮浓度稀释曲线做综合性的比 
表 4 相对籽粒产量(RY)的 RMSE, $n-R M S E$ 和 $R^{2}$ 的值(根据 2016-2017 年不同时期的氮营养指数(NNI)和累积氮亏(AND)预测)

Table 4 Values of RMSE, $n$-RMSE and $R^{2}$ for prediction of relative yield (RY) from nitrogen nutrition index (NNI), and accumulated nitrogen deficit (AND), respectively, at different growth stages using data obtained in 2016 and 2017

\begin{tabular}{|c|c|c|c|c|c|c|c|c|c|c|c|c|}
\hline \multirow{2}{*}{$\begin{array}{c}\text { 部位 } \\
\text { Organ or plant }\end{array}$} & \multirow{2}{*}{$\begin{array}{c}\text { 品种 } \\
\text { Cultivar }\end{array}$} & \multirow{2}{*}{$\begin{array}{c}\text { 参数 } \\
\text { Parameter }\end{array}$} & \multicolumn{5}{|c|}{ 氮亏缺 Accumulated nitrogen deficit (AND) } & \multicolumn{5}{|c|}{ 氮营养指数 Nitrogen nutrition index (NNI) } \\
\hline & & & $\mathrm{V} 3$ & V6 & V8 & V12 & VT & V3 & V6 & V8 & V12 & VT \\
\hline 叶片 & 正大 12 & RMSE & 0.08 & 0.08 & 0.09 & 0.03 & 0.05 & 0.08 & 0.12 & 0.08 & 0.05 & 0.05 \\
\hline \multirow[t]{5}{*}{ Leaf } & Zhengda 12 & $n$-RMSE & 9.10 & 9.04 & 10.41 & 3.51 & 5.23 & 8.85 & 13.65 & 8.39 & 5.72 & 5.31 \\
\hline & & $R^{2}$ & 0.90 & 0.89 & 0.86 & 0.98 & 0.96 & 0.90 & 0.76 & 0.91 & 0.95 & 0.96 \\
\hline & 陕单 609 & RMSE & 0.11 & 0.06 & 0.08 & 0.05 & 0.05 & 0.08 & 0.07 & 0.07 & 0.03 & 0.05 \\
\hline & Shaandan 609 & $n$-RMSE & 12.40 & 6.86 & 8.69 & 5.81 & 5.40 & 8.80 & 8.12 & 7.90 & 2.82 & 5.11 \\
\hline & & $R^{2}$ & 0.83 & 0.93 & 0.88 & 0.95 & 0.95 & 0.88 & 0.90 & 0.90 & 0.98 & 0.96 \\
\hline 茎 & 正大 12 & RMSE & 0.11 & 0.10 & 0.10 & 0.06 & 0.06 & 0.08 & 0.10 & 0.06 & 0.06 & 0.06 \\
\hline \multirow[t]{5}{*}{ Stem } & Zhengda 12 & $n$-RMSE & 11.83 & 10.66 & 10.96 & 7.16 & 6.67 & 8.81 & 11.40 & 6.46 & 6.79 & 6.49 \\
\hline & & $R^{2}$ & 0.83 & 0.85 & 0.84 & 0.93 & 0.94 & 0.90 & 0.82 & 0.94 & 0.94 & 0.94 \\
\hline & 陕单 609 & RMSE & 0.10 & 0.10 & 0.06 & 0.04 & 0.04 & 0.06 & 0.08 & 0.05 & 0.04 & 0.03 \\
\hline & Shaandan 609 & $n$-RMSE & 11.00 & 11.12 & 6.35 & 3.92 & 4.18 & 6.46 & 9.14 & 5.57 & 4.74 & 3.16 \\
\hline & & $R^{2}$ & 0.87 & 0.81 & 0.94 & 0.97 & 0.97 & 0.93 & 0.87 & 0.95 & 0.96 & 0.98 \\
\hline 植株 & 正大 12 & RMSE & 0.15 & 0.09 & 0.07 & 0.06 & 0.04 & 0.10 & 0.09 & 0.06 & 0.06 & 0.04 \\
\hline \multirow[t]{5}{*}{ Plant } & Zhengda 12 & $n-\mathrm{RMSE}$ & 17.09 & 9.87 & 7.81 & 6.11 & 4.63 & 10.70 & 9.97 & 7.03 & 6.57 & 4.38 \\
\hline & & $R^{2}$ & 0.65 & 0.88 & 0.92 & 0.95 & 0.97 & 0.86 & 0.88 & 0.94 & 0.94 & 0.97 \\
\hline & 陕单 609 & RMSE & 0.12 & 0.10 & 0.08 & 0.04 & 0.07 & 0.08 & 0.09 & 0.04 & 0.03 & 0.04 \\
\hline & Shaandan 609 & $n$-RMSE & 13.47 & 11.25 & 8.32 & 4.65 & 7.85 & 9.24 & 9.42 & 4.91 & 3.03 & 4.93 \\
\hline & & $R^{2}$ & 0.80 & 0.86 & 0.92 & 0.97 & 0.93 & 0.91 & 0.90 & 0.97 & 0.99 & 0.97 \\
\hline
\end{tabular}

V3、V6、V8、V12、VT 和 R6 分别代表玉米的三叶期、六叶期(拔节期)、八叶期、十二叶期(大喇叭口期)、抽雄期和完熟期。

V3, V6, V8, V12, VT, and R6 represent the third, sixth, eighth, twelfth leaf stages, tasseling, and physiological maturity stages, respectively.

较。在临界氮浓度稀释曲线中，参数 $a$ 代表单位生 物量氮浓度; 参数 $b$ 代表临界氮浓度, 随地上部干 物质的增加而递减。与前人研究结果相比(表 6), 植 株临界氮浓度曲线的结果接近陕西关中地区参数 $a$ 的范围 2.14 2.25, 参数 $b$ 的范围 0.14 0.31, 这 2 个 参数的值低于已有的其他模型 ${ }^{[37-38]}$, 进一步说明已 有模型在关中地区不适宜，需要建立属于本生态环 境下全面的临界氮浓度稀释曲线。2 个品种植株临 界氮浓度稀释曲线 $a$ 值存在显著差异, 说明参数 $a$ 受到品种的影响。强生才等 ${ }^{[28]}$ 对不同降雨年型下植 株临界氮浓度曲线的建立指出参数 $b$ 会随降雨年型 的改变而改变。本研究结果进一步补充了该地区模 型在玉米品种建立上的缺失。同一个试验中基于玉 米不同部位建立的临界氮浓度稀释曲线表明，基于 LDM 临界曲线参数 $b$ 值最小, 说明叶片作为主要 代谢器官的重要生理作用, 这一结果与在水稻研究 中 ${ }^{[22]}$ 的结果一致。同时, 基于 SDM 的临界氮浓度稀 释曲线参数 $b$ 大于基于 LDM 的临界氮浓度稀释曲 线的 $b$ 值, 这表明玉米的茎氮浓度低于叶片氮浓度, 而其稀释速率高于叶片的稀释速率，这种差异主要
是由于茎叶比引起的, 营养生长期间玉米叶片作为 主要的光合器官, 大量的氮素从植物的结构组分(茎) 转运到代谢组分(叶)以需要维持一定的氮浓度保证 光合作用运行, 导致叶片氮浓度的缓慢下降 ${ }^{[39]}$ 。在 玉米不同器官氮积累变化的研究中发现, 叶片氮浓 度始终高于茎, 并且随着干物质转运过程的发生而 变化, 从而造成了茎氮浓度稀释速率高于叶片 ${ }^{[40-41]}$ 。 基于小麦和水稻 LDM 的临界氮浓度稀释曲线也得 出了几乎相似的 $b$ 值 ${ }^{[14,23]}$ 。同时氮高效品种与氮低 效品种叶片参数 $a$ 和 $b$ 值相似, 氮高效品种茎和植 株的参数 $a$ 值低于氮低效品种, 而茎和植株的参数 $b$ 值高于氮低效品种。与不同玉米品种研究结果相似, $a$ 和 $b$ 值的变化方向是一致 ${ }^{[42]}$, 本试验结果进一步 表明，造成氮效率的差异主要来自于茎的差异而不 是叶片。这可能反映了氮高效品种的茎中氮素高的 稀释速率，一方面与其干物质的快速积累有关，另 一方面反映出氮素在不同器官的分配，氮高效品种 在营养生长阶段高的茎氮素稀释速率，保证转运到 叶片更多氮素以维持光合作用, Chen 等 ${ }^{[43]}$ 的结果指 出, 与氮低效品种相比, 氮高效品种具有较高的茎 


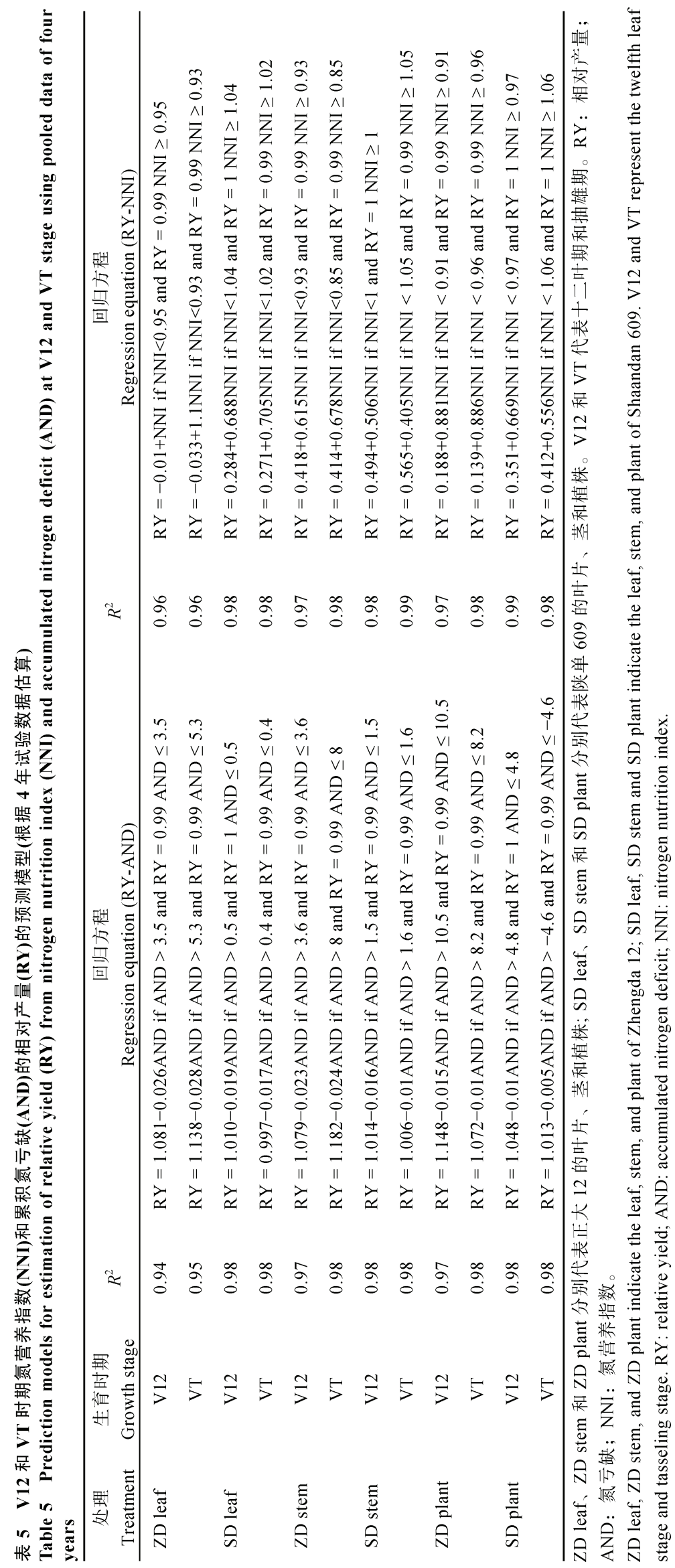


表 6 基于不同部位建立的临界氮浓度稀释曲线与其他模型参数的比较

Table 6 Comparison of parameters of critical N dilution curves based on different organs and other model parameters

\begin{tabular}{|c|c|c|c|c|c|}
\hline $\begin{array}{l}\text { 部位 } \\
\text { Organ }\end{array}$ & $\begin{array}{l}\text { 作物 } \\
\text { Crop }\end{array}$ & $\begin{array}{l}\text { 地点 } \\
\text { Site }\end{array}$ & $a$ & $b$ & $\begin{array}{l}\text { 参考文献 } \\
\text { Reference }\end{array}$ \\
\hline 植株 & 玉米 Maize & 关中平原 Guanzhong Plain & 2.25 & 0.27 & Li Z P et al. ${ }^{[29]}$ \\
\hline \multirow[t]{4}{*}{ Plant } & & 黄淮海平原 Huanghuaihai Plain & 3.34 & 0.396 & Liang X G et al. ${ }^{[38]}$ \\
\hline & & 华北平原 North China Plain & 2.72 & 0.27 & Yue S C et al. ${ }^{[37]}$ \\
\hline & & 本试验 This study & 2.47 & 0.24 & \\
\hline & & & 2.33 & 0.26 & \\
\hline 叶片 & 玉米 Maize & 黄淮海平原 Huanghuaihai Plain & 3.45 & 0.22 & Zhao B et al. ${ }^{[10]}$ \\
\hline \multirow[t]{5}{*}{ Leaf } & 水稻 Rice & 长江中下游平原 the Middle-Lower Yangtze Plains & 3.76 & 0.22 & Wang X L ${ }^{[44]}$ \\
\hline & 小麦 Wheat & 长江中下游平原 the Middle-Lower Yangtze Plains & 3.06 & 0.15 & Yao X et al..$^{[23]}$ \\
\hline & & 关中平原 Guanzhong Plain & 3.96 & 0.14 & Qiang S C et al. ${ }^{[45]}$ \\
\hline & & 本试验 This study & 2.64 & 0.20 & \\
\hline & & & 2.61 & 0.21 & \\
\hline 茎 & 小麦 Wheat & 长江中下游平原 the Middle-Lower Yangtze Plains & 2.50 & 0.44 & Wang X L ${ }^{[44]}$ \\
\hline \multirow[t]{3}{*}{ Stem } & 水稻 Rice & 长江中下游平原 the Middle-Lower Yangtze Plains & 2.26 & 0.32 & Ata-Ul-Karim S T et al. ${ }^{[22]}$ \\
\hline & & 本试验 This study & 1.58 & 0.39 & \\
\hline & & & 1.83 & 0.34 & \\
\hline
\end{tabular}

$a$ 和 $b$ 为模型参数。 $a$ and $b$ are the model parameters.

的氮转运效率。氮高效品种干物质及氮素在各器官 的分配使得其能够在较低的氮素需求下实现高产。 玉米基于茎干物质建立的临界氮浓度稀释曲线仍是 一个空白，本试验证明基于 SDM 的临界氮浓度稀释 曲线可以用于玉米氮营养诊断, 参数 $a$ 比其他作物 (小麦和水稻)要小, 这也是造成本地区整株植株水 平参数 $a$ 偏低的原因 ${ }^{[37]}$, 参数 $b$ 与其他作物上相似, 对于不同的氮效率品种来说, $b$ 值差异显著, 可以用 于品种间的氮营养诊断。

\section{2 对夏玉米氮素诊断和作物建模的意义}

建立基于 LDM、SDM 和 PDM 的临界氮浓度稀 释曲线的主要目的是用农学的研究方法诊断作物的 氮营养状态，通过临界氮浓度稀释曲线来诊断夏玉 米营养生育阶段的氮素盈亏状态，建立与产量的模 型 ${ }^{[46]}$ 从而对产量进行预测。同时, Zhao 等 ${ }^{[34]}$ 提出开 花前玉米的氮素状态与花后穗部显著相关, 所以如 果在开花前能很好的诊断玉米氮素状况对于花后施 肥和成熟期产量的预测具有一定的预见性。在达到 最佳氮肥水平前, 夏玉米的 NNI 和籽粒产量逐渐增 加。本研究中的叶片 NNI 值的范围为 $0.66 \sim 1.14$, 茎 $\mathrm{NNI}$ 值的范围为 $0.50 \sim 1.28$, 植株 NNI 值为 $0.62 \sim 1.20$ 。 各部位 NNI 值达到 1 左右时, 产量不再增加。Ziadi 等 ${ }^{[17]}$ 确定了当玉米的 NNI 低于 0.93 时, NNI 与 RY 之间呈正相关。基于植株 NNI 与产量的关系显示了 使用 NNI 进行估算作物氮需求、作物氮状况和籽粒
产量潜力 ${ }^{[6-7,13-14,47]}$ 的可行性。V12-VT 时期是考虑 玉米追施氮肥的关键时期，并且在这阶段建立的模 型具有很强的稳定性。与水稻相比 ${ }^{[22]}$, 追肥相对靠 后, Zhao 等 ${ }^{[34]}$ 指出玉米更依赖于开花后氮素的状况, 而小麦更依赖于花前氮的状况来满足穗部的生长, 这可能由于, 相比 $\mathrm{C}_{3}$ 作物, $\mathrm{C}_{4}$ 作物高的光合氮效率 使得其可以利用低的氮素来获得高的产量。V12-VT 阶段的 RY 与 NNI 和 RY 与 AND 之间的关系准确地 解释了在受氮素限制和不受氮素限制生长条件下 $\mathrm{RY}$ 的变化。因此, 这些阶段可以作为产量预测模型 的最佳时期。相反，早期营养生长过程中 RY 与 NNI 和 RY 与 AND 之间的关系, 稳定性相对较差, 因此 预测玉米产量的准确性较差。造成稳定性较差的原 因, 一方面, 在作物早期生长阶段, 茎叶比的变化 比后期生长阶段变化更快，玉米苗期到拔节后期的 茎叶比和氮浓度差异较大 ${ }^{[10]}$, 另一方面, 大田条件 下, 玉米生长不均匀难以评估玉米的生长阶段。这 些都会影响基于生长阶段的临界氮浓度稀释曲线对 植物氮诊断的准确性。基于不同部位的临界氮浓度 稀释曲线的 NNI 和 AND 可以对 RY 进行准确地预测, 顺应精准农业对玉米产量的实时估测提出的要求, 所以上述模型关系可以用于估计玉米籽粒产量和对 管理决策的制定。以上提出的诊断方法为作物模型 参数的调整优化具有很大的借鉴, 以便在不同的气 候条件、基因型和管理实践下应用。这些将有助于 
提供对作物生长和产量的准确预测，为生产和管理 决策提供支持。这种方法的应用可以帮助根据不同 的氮肥施用量设定产量目标。除了破坏性采样方法 外, 叶绿素仪和冠层反射率等方法还可以用于 NNI 的快速和非破坏性估计 ${ }^{[7,48-49]}$ 。因此, RY 和 NNI 之 间的关系的建立对于玉米产量的估算具有实际意义 [17]。在本研究中，关键生育时期，基于不同部位的临 界氮浓度稀释曲线的 NNI 与 RY 和 AND 与 RY 的关 系，可以看出在 V12-VT 时期基于 LDM、SDM 和 PDM 建立的临界氮浓度稀释曲线的 NNI 和 AND 对 $\mathrm{RY}$ 的预测是可靠的。鉴于该模型可准确地探测作物 氮素营养状况，因此，氮营养指数和氮素亏缺模型 可以应用于精准农业变量施肥中。

总而言之, 本研究表明, 基于不同部位的临界 氮浓度稀释曲线，玉米在关键生长阶段 RY 与 NNI 和 AND 的关系可以用于估算籽粒产量。特别是在 V12-VT 阶段的关系准确地解释了在受氮素限制和 不受氮素限制生长条件下 $\mathrm{RY}$ 的偏差, 并且可靠地 用于预测籽粒产量。此外，利用 V12-VT 阶段的基 于不同部位的临界氮浓度稀释曲线预测籽粒产量与 玉米生产系统中追施氮肥的时间是同步的。因此， 新建立的模型的实施不仅可以预测玉米产量潜力, 而且有助于玉米生产中精确的氮素管理。为了保证 模型更加广泛地应用, 需要对不同气候, 水分和耕 作条件下进行模型建立和验证。

\section{4 结论}

本研究首次采用不同部位指标建立和比较评价 了玉米临界氮浓度稀释曲线。使用独立的试验数据 验证了 3 种临界氮浓度稀释曲线都是可靠的。基于 LDM、SDM 和 PDM 建立的临界氮浓度稀释曲线对 $\mathrm{RY}$ 的预测是稳定。因此, 出于成本的考虑, 在一定 的条件下，可以用基于 LDM 和 SDM 建立的临界氮 浓度稀释曲线可以取代基于 PDM 建立的临界氮浓 度稀释曲线对玉米的氮素进行诊断和产量预测。在 受氮素限制条件下，玉米基于不同部位计算的 NNI 与产量构成之间显著正相关。这项研究表明，基于 不同部位的临界氮浓度稀释曲线的关键作物生长阶 段的 RY-NNI 和 RY-AND 关系可以用于为玉米适当 的氮肥施用量做出指导。特别是, V12-VT 阶段的稳 定关系准确地解释了氮素缺乏和最佳生长条件下 $R Y$ 和 NNI 与 RY 和 AND 的变化, 并且可以可靠地 对 RY 进行预测, 从而量化了开花前氮肥的施用量。

\section{References}

[1] Erisman J W, Sutton M A, Galloway J, Klimont Z, Winiwarter W. How a century of ammonia synthesis changed the world. Nat Geosci, 2008, 1: 636-639.

[2] 王西娜, 王朝辉, 李生秀. 施氮量对夏季玉米产量及土壤水氮 动态的影响. 生态学报, 2007, 27(1): 197-204.

Wang X N, Wang Z H, Li S X. The effect of nitrogen fertilizer rate on summer maize yield and soil water nitrogen dynamics. Acta Ecol Sin, 2007, 27(1): 197-204 (in Chinese with English abstract).

[3] Ramanantenasoa M M J, Génermont S, Gilliot J M, Makowski D. Metamodeling methods for estimating ammonia volatilization from nitrogen fertilizer and manure applications. $J$ Environ Manag, 2019, 236: 195-205.

[4] Galloway J N, Cowling E B. Reactive nitrogen and the world: 200 years of change. Ambio, 2002, 3: 64-71.

[5] 宁芳, 张元红, 温鹏飞, 王瑞, 王倩, 董朝阳, 贾广灿, 李军. 不同降水状况下旱地玉米生长与产量对施氮量的响应. 作物 学报, 2019, 45: 777-791.

Ning F, Zhang Y H, Wen P F, Wang R, Wang Q, Dong Z Y, Jia G $\mathrm{C}$, Li J. Responses of maize growth and yield to nitrogen application in dryland under different precipitation conditions. Acta Agron Sin, 2019, 45: 777-791 (in Chinese with English abstract).

[6] Zhao B, Ata-Ul-Karim S T, Liu Z D, Zhang J Y, Xiao J F, Liu Z G, Qin A Z, Ning D F, Yang Q X, Zhang Y H, Duan A W. Simple assessment of nitrogen nutrition index in summer maize by using chlorophyll meter readings. Front Plant Sci, 2018, 9: 11.

[7] Zhao B, Liu Z D, Ata-Ul-Karim S T, Xiao J F, Liu Z G, Qi A Z, Ning D F, Nan J Q, Duan A W. Rapid and nondestructive estimation of the nitrogen nutrition index in winter barley using chlorophyll measurements. Field Crops Res, 2016, 185: 59-68.

[8] Justes E, Mary B, Meynard J M, Machet J M, Thelier-Huche L. Determination of A critical nitrogen dilution curve for winter-wheat Crops. Ann Bot, 1994, 74: 397-407.

[9] Ata-Ul-Karim S T, Yao X, Liu X J, Cao W X, Zhu Y. Development of critical nitrogen dilution curve of japonica rice in Yangtze River Reaches. Field Crops Res, 2013, 149: 149-158.

[10] Zhao B, Ata-Ul-Karim S T, Liu Z, Ning D F, Xiao J F, Liu Z G, Qin A Z, Nan J Q, Duan A W. Development of a critical nitrogen dilution curve based on leaf dry matter for summer maize. Field Crops Res, 2017, 208: 60-68.

[11] Wang X L, Ye T Y, Ata-Ul-Karim S T, Zhu Y, Liu L L, Cao W X, Tang L. Development of a critical nitrogen dilution curve based on leaf area duration in wheat. Front Plant Sci, 2017, 8: 1517.

[12] 付江鹏, 贺正, 贾彪, 刘慧芳, 李振洲, 刘志. 滴灌玉米临界 氮稀释曲线与氮素营养诊断研究. 作物学报, 2020, 46: 290-299.

Fu J P, He Z, Jia B, Liu H F, Li Z Z, Liu Z. Critical nitrogen dilution curve and nitrogen nutrition diagnosis of maize with drip irrigation. Acta Agron Sin, 2020, 46: 290-299 (in Chinese with English abstract).

[13] Ata-Ul-Karim S T, Liu X J, Lu Z Z, Yuan Z F, Zhu Y, Cao W X. In-season estimation of rice grain yield using critical nitrogen dilution curve. Field Crops Res, 2016, 195: 1-8.

[14] Ata-Ul-Karim S T, Liu X J, Lu Z Z, Zheng H B, Cao W X, Zhu Y. 
Estimation of nitrogen fertilizer requirement for rice crop using critical nitrogen dilution curve. Field Crops Res, 2017, 201: 32-40.

[15] 岳松华, 刘春雨, 黄玉芳, 叶优良. 豫中地区冬小麦临界氮稀 释曲线与氮营养指数模型的建立. 作物学报, 2016, 42: 909-916.

Yue S H, Liu C Y, Huang Y F, Ye Y L. Simulating critical nitrogen dilution curve and modeling nitrogen nutrition index in winter wheat in central Henan area. Acta Agron Sin, 2016, 42: 909-916 (in Chinese with English abstract).

[16] Yao Y K, Miao Y X, Cao Q, Wang H Y, Gnyp M L, Bareth G, Khosla R, Yang W, Liu F Y, Liu C. In-season estimation of rice nitrogen status with an active crop canopy sensor. IEEE J-Stars, 2014, 7: 4403-4413.

[17] Ziadi N, Bélanger G, Claessens A, Lefebvre L, Cambouris A N, Tremblay N, Nolin M C, Parent L E. Determination of a critical nitrogen dilution curve for spring wheat. Agron J, 2010, 102: 241-250.

[18] Lemaire G, Jeuffroy M H, Gastal F. Diagnosis tool for plant and crop N status in vegetative stage: Theory and practices for crop N management. Eur J Agron, 2008, 28: 614-624.

[19] Zhang K, Yuan Z F, Yang T C, Lu Z Z, Cao Q, Tian Y C, Zhu Y, Cao W X, Liu X J. Chlorophyll meter-based nitrogen fertilizer optimization algorithm and nitrogen nutrition index for in-season fertilization of paddy rice. Agron J, 2020, 112: 1-13.

[20] Ziadi N, Brassard M, Bélanger G, Claessens A, Tremblay N, Cambouris A, Nolin M, Parent L E. Chlorophyll measurements and nitrogen nutrition index for the evaluation of corn nitrogen status. Agron J, 2008, 100: 271-273.

[21] Ata-Ul-Karim S T, Yao X, Liu X J, Cao W X, Zhu Y. Determination of critical nitrogen dilution curve based on stem dry matter in rice. PLoS One, 2014, 9: e104540.

[22] Ata-Ul-Karim S T, Zhu Y, Liu X J, Cao Q, Tian Y C, Cao W X. Comparison of different critical nitrogen dilution curves for nitrogen diagnosis in rice. Sci Rep, 2017, 7: 42679.

[23] Yao X, Zhao B, Tian Y C, Liu X J, Ni J, Cao W X, Zhu Y. Using leaf dry matter to quantify the critical nitrogen dilution curve for winter wheat cultivated in eastern China. Field Crops Res, 2014, 159: 33-42.

[24] Huang S Y, Miao Y X, Cao Q, Yao Y K, Zhao G M, Yu W F, Shen J N, Yu K, Bareth G. A new critical nitrogen dilution curve for rice nitrogen status diagnosis in Northeast China. Pedosphere, 2018, 28: 814-822.

[25] Zhao B, Yao X, Tian Y C, Liu X J, Ata-Ul-Karim S T, Ni J, Cao W X, Zhu Y. New critical nitrogen curve based on leaf area index for winter wheat. Agron J, 2014, 106: 379-383.

[26] Zhao B, Ata-Ui-Karim S T, Yao X, Tian Y C, Cao W X, Zhu Y, Liu X J. A new curve of critical nitrogen concentration based on spike dry matter for winter wheat in Eastern China. PLoS One, 2016, 11: e164545.

[27] Zhao Z G, Wang E L, Wang Z M, Zang H Z, Liu Y P, Angus J F. A reappraisal of the critical nitrogen concentration of wheat and its implications on crop modeling. Field Crops Res, 2014, 164: 65-73.

[28] 强生才, 张富仓, 向友珍, 张燕, 问世程, 邢英英. 关中平原 不同降雨年型夏玉米临界氮稀释曲线模拟及验证. 农业工程
学报, 2015, 31(17): 168-175.

Qiang S C, Zhang F C, Xiang Y Z, Zhang Y, Yan S C, Xing Y Y. Simulation and validation of critical nitrogen dilution curve for summer maize in Guanzhong Plain during different rainfall years. Trans CSAE, 2015, 31(17): 168-175 (in Chinese with English abstract).

[29] 李正鹏, 冯浩, 宋明丹. 关中平原冬小麦临界氮浓度稀释曲线 和氮营养指数研究. 农业机械学报, 2015, 46(10): 177-183.

Li Z P, Feng H, Song M D. Development and validation of critical nitrogen content curve for maize in Guanzhong area. Trans CSAM, 2015, 46(10): 135-141 (in Chinese with English abstract).

[30] Bremner J M, Mulvancy C S. Nitrogen-total. In: Page A L, Miller R H, Keeney D R, eds. Methods of Soil Analysis. Madison: American Society of Agronomy, 1982. pp 595-624.

[31] Yang J, Greenwood D J, Rowell D L, Wadsworth G A, Burns I G. Statistical methods for evaluating a crop nitrogen simulation model, N_ABLE. Agric Syst, 2000, 64: 37-53.

[32] Jamieson P D, Porter J R, Wilson D R. A test of the computer simulation model ARCWHEAT1 on wheat crops grown in New Zealand. Field Crops Res, 1991, 27: 337-350.

[33] He Z Y, Qiu X L, Ata-Ul-Karim S T, Li Y D, Liu X J, Cao Q, Zhu Y, Cao W X, Tang L. Development of a critical nitrogen dilution curve of double cropping rice in south China. Front Plant Sci, 2017, 8: 638.

[34] Zhao B, Niu X L, Ata-Ul-Karim S T, Wang L G, Duan A W, Liu $\mathrm{Z}$ D, Lemaire G. Determination of the post-anthesis nitrogen status using ear critical nitrogen dilution curve and its implications for nitrogen management in maize and wheat. Eur J Agron, 2020, 113: 125967.

[35] Song L G, Wang S, Ye W J. Establishment and application of critical nitrogen dilution curve for rice based on leaf dry matter. Agron J, 2020, 10: 367.

[36] 梁效贵, 张经廷, 周丽丽, 李旭辉, 周顺利. 华北地区夏玉米 临界氮稀释曲线和氮营养指数研究. 作物学报, 2013, 39: 292-299.

Liang X G, Zhang J T, Zhou L L, Li X H, Zhou S L. Critical nitrogen dilution curve and nitrogen nutrition index for summer maize in north China plain. Acta Agron Sin, 2013, 39: 292-299 (in Chinese with English abstract).

[37] Yue S C, Sun F L, Meng Q F, Zhao R F, Li F, Chen X P, Zhang F $\mathrm{S}$, Cui Z L. Validation of a critical nitrogen curve for summer maize in the North China Plain. Pedosphere, 2014, 24: 76-83.

[38] Liang X G, Zhang Z L, Zhou L L, Shen S, Gao Z, Zhang L, Lin S, Pan Y Q, Zhou S L. Localization of maize critical N curve and estimation of NNI by Chlorophyll. Int J Plant Prod, 2018, 12: 85-94.

[39] Novoa R, Loomis R. Nitrogen and plant production. Plant Soil, 1981, 58: 177-204.

[40] 侯有良, Brien L O, 钟改荣. 小麦不同器官氮素累积分布动态 规律的研究. 作物学报, 2001, 27: 493-499.

Hou Y L, Brien L O, Zhong G R. Study on the dynamic change of the distribution and accumulation of nitrogen in different plant parts of wheat. Acta Agron Sin, 2001, 27: $493-499$ (in Chinese with English abstract).

[41] Sinclair T R, Horie T. Leaf nitrogen, photosynthesis and crop radiation use efficiency: a review. Crop $S c i, 1989,29$ : 90-98. 
[42] 安志超, 黄玉芳, 汪洋, 赵亚南, 岳松华, 师海斌, 叶优良. 植 物营养与肥料学报, 2019, 25: 123-133.

An Z C, Huang Y F, Wang Y, Zhao Y N, Yue S H, Shi H B, Ye Y L. Critical nitrogen concentration dilution model and nitrogen nutrition diagnosis in summer maize with different nitrogen efficiencies. J Plant Nutr Fert, 2019, 25: 123-133 (in Chinese with English abstract).

[43] Chen Y L, Xiao C X, Chen X C, Li Q, Zhang J, Chen F J, Yuan L $\mathrm{X}$, Mi G H. Characterization of the plant traits contributed to high grain yield and high grain nitrogen concentration in maize. Field Crops Res, 2014, 159: 1-9.

[44] 王晓玲. 长江中下游稻麦两熟区冬小麦植株器官临界氮浓度 模型构建及氮素诊断调控研究. 南京农业大学博士学位论文, 江苏南京, 2017.

Wang X L. Study on Construction Critical Nitrogen Concentration Dilution Models Based on Plant Organs and Diagnosis and Regulation of Wheat in the Middle and Lower Reaches of the Yangtze River. PhD Dissertation of Graduate School of Nanjing Agricultural University, Nanjing, Jiangsu, China, 2017 (in Chinese with English abstract).

[45] 强生才, 张富仓, 田建柯, 吴悠, 问世程, 范军亮. 基于叶片 干物质的冬小麦临界氮浓度稀释曲线模拟研究. 农业机械学 报, 2015, 46(11): 121-128.
Qiang S C, Zhang F C, Tian J K, Wu Y, Yan S C, Fan J L. Deve lopment of critical nitrogen dilution curve in winter wheat based on leaf dry matter. Trans CSAM, 2015, 46(11): 121-128 (in Chinese with English abstract).

[46] Stockle C O, Debaeke P. Modeling crop nitrogen requirements: a critical analysis. Eur J Agron, 1997, 7: 161-169.

[47] 刘朋召, 师祖姣, 宁芳, 王瑞, 王小利, 李军. 不同降雨状况 下渭北旱地春玉米临界氮稀释曲线与氮素营养诊断. 作物学 报, 2020, 46: 1225-1237.

Liu P Z, Shi Z J, Ning F, Wang R, Wang X L, Li J. Critical nitrogen dilution curves and nitrogen nutrition diagnosis of spring maize under different precipitation patterns in Weibei dryland. Acta Agron Sin, 2020, 46: 1225-1237 (in Chinese with English abstract).

[48] Yuan Z F, Ata-Ul-Karim S T, Cao Q, Lu Z Z, Cao W X, Zhu Y, Liu X J. Indicators for diagnosing nitrogen status of rice based on chlorophyll meter readings. Field Crops Res, 2016, 185: $12-20$

[49] Zhao B, Duan A W, Ata-Ul-Karim S T, Liu Z D, Chen Z F, Gong Z H, Zhang J Y, Xiao J F, Liu Z G, Qin A Z, Ning D F. Exploring new spectral bands and vegetation indices for estimating nitrogen nutrition index of summer maize. Eur J Agron, 2018, 93: $113-125$. 